\title{
COMPLETE $(p, q)$-ELLIPTIC INTEGRALS WITH APPLICATION TO A FAMILY OF MEANS
}

\author{
TOSHIKI KAMIYA AND SHINGo TAKEUCHI
}

Abstract. The complete elliptic integrals are generalized by using the generalized trigonometric functions with two parameters. As an application of the integrals, an alternative proof of a result for a family of means by Bhatia and $\mathrm{Li}$, which involves the logarithmic mean and the arithmetic-geometric mean, is given. Moreover, it is shown that a particular relation holds for the generalized integrals.

Mathematics subject classification (2010): 33E05, 33C75, 34L10.

Keywords and phrases: complete elliptic integrals, generalized trigonometric functions, arithmeticgeometric mean, logarithmic mean, Gaussian hypergeometric functions, $p$-Laplacian.

\section{REFERENCES}

[1] G. Andrews, R. Askey And R. Roy, Special functions, Encyclopedia of Mathematics and its Applications, 71. Cambridge University Press, Cambridge, 1999.

[2] R. Bhatia And R.-C. Li, An interpolating family of means, Commun. Stoch. Anal. 6 (2012), no. 1, 15-31.

[3] B.A. Bhayo AND L. Yin, On generalized $(p, q)$-elliptic integrals, preprint, arXiv:1507.00031.

[4] J.M. Borwein And P.B.Borwein, Pi and the AGM, A study in analytic number theory and computational complexity. Reprint of the 1987 original. Canadian Mathematical Society Series of Monographs and Advanced Texts, 4. A Wiley-Interscience Publication. John Wiley \& Sons, Inc., New York, 1998

[5] P. DRÁBEK AND R. MANÁSEVICH, On the closed solution to some nonhomogeneous eigenvalue problems with p-Laplacian, Differential Integral Equations 12 (1999), 773-788.

[6] J.LANG AND D.E. EDMUNDS, Eigenvalues, embeddings and generalised trigonometric functions, Lecture Notes in Mathematics, 2016. Springer, Heidelberg, 2011.

[7] P. LindQVist, Some remarkable sine and cosine functions, Ricerche Mat. 44 (1995), no. 2, 269-290.

[8] N. NAKAMURA, Order relations among some interpolating families of means, Toyama Math. J. 35 (2012), 35-48.

[9] S. TAKEUCHI, Generalized Jacobian elliptic functions and their application to bifurcation problems associated with p-Laplacian, J. Math. Anal. Appl. 385 (2012), no. 1, 24-35.

[10] S. TAKeUChI, The basis property of generalized Jacobian elliptic functions, Commun. Pure Appl. Anal. 13 (2014), no. 6, 2675-2692.

[11] S. TAKEUCHI, A new form of the generalized complete elliptic integrals, Kodai Math. J. 39, no. 1 (2016), 202-226.

[12] S. TAKEUCHI, Legendre-type relations for generalized complete elliptic integrals, J. Class. Anal. 9 , no. 1 (2016), 35-42.

[13] L. YIN AND L.-G.HUANG, Inequalities for the generalized trigonometric and hyperbolic functions with two parameters, J. Nonlinear Sci. Appl. 8 (2015), no. 4, 315-323. 